\title{
Estimation of gamma-glutamyl transferase as a suitable simple biomarker of the cardiovascular risk in children with non-alcoholic fatty liver disease
}

\author{
Anna Bobrus-Chociej ${ }^{\bowtie}$, Urszula Daniluk¹, Marta Flisiak-Jackiewicz', Małgorzata Wojtkowska², \\ Monika Kłusek-Oksiuta ${ }^{1}$, Eugeniusz Tarasów ${ }^{3}$ and Dariusz Lebensztejn ${ }^{1}$
}

'Departement of Pediatrics, Gastroenterology, Hepatology, Nutrition and Allergology; ${ }^{2}$ Department of Pediatric Radiology; ${ }^{3}$ Department of Radiology, Medical University of Bialystok, Białystok, Poland

Non-alcoholic fatty liver disease (NAFLD) is a hepatic manifestation of metabolic syndrome and a risk factor for cardiovascular disease (CVD). Research conducted in adults has proven that GGT can also be an independent risk factor for CVD. The aim of this study was to ascertain if GGT can be regarded as a simple biomarker of cardiovascular risk in obese children with NAFLD. One hundred obese children, aged 7-17 years, with suspected liver pathology were admitted to our Department. Viral hepatitis and autoimmune, toxic and selected metabolic liver diseases were excluded. Anthropometry, laboratory tests, 'HMR spectroscopy and evaluation of the common carotid artery intima-media thickness (IMT) were performed in all subjects. NAFLD was confirmed in 38 obese patients. There was a significantly higher activity of GGT and ALT, the concentration of total and LDL cholesterol, waist circumference, left coronary artery IMT, mean IMT value and total lipids in 'HMRS in children with NAFLD in comparison to non-hepatopathic obese children. Logistic regression analysis indicated that GGT, total cholesterol, LDL-cholesterol, left IMT and waist circumference significantly affected the development of NAFLD in obese children. In ROC analysis only GGT, waist circumference and left IMT allowed to differentiate children with NAFLD from those without steatosis with GGT having the highest result (AUC=0.94). GGT activity in patients revealed weak or at the upper limit of statistical significance correlation with traditional cardiovascular risk factors: glucose level, waist circumference, BMI, total cholesterol, LDL-cholesterol and insulin level. This allows to suggest, that GGT might be a potential reliable, simple and non-invasive biochemical marker for estimation of cardiovascular risk in obese children with NAFLD. However, further studies on larger population are necessary to confirm that observation.

Key words: non-alcoholic fatty liver disease, children, obesity, GGT, cardiovascular risk

Received: 07 May, 2018; revised: 05 September, 2018; accepted: 14 September, 2018; available on-line: 31 October, 2018

e-mail: anna.chociej@op.pl

Abbreviations: ALT, alanine aminotransferase; BMI, body mass index; CVD, cardiovascular disease; GGT, gamma-glutamyl transferase; HDL, high-density lipoprotein; 'HMRS, proton magnetic resonance spectroscopy; HOMA-IR, homeostasis model assessment-insulin resistance; hsCRP, high sensitive C-reactive protein; IMT, intima-media thickness; LDL, low-density lipoprotein; NAFLD, non-alcoholic fatty liver disease; MRI, magnetic resonance imaging; $\mathrm{ROC}$, receiver operating characteristics

\section{INTRODUCTION}

With the global epidemic of obesity, non-alcoholic fatty liver disease (NAFLD) has become the most common chronic liver disorder not only among adults in the Western world, but also in children and adolescents, affecting approximately $10-20 \%$ of the general pediatric population. According to the current data, the prevalence of NAFLD in overweight and obese youths is about $70 \%$, compared to $7 \%$ in those of normal weight (Temple et al., 2016). The incidence of NAFLD has been increasing in both developed and developing countries (Marchesini et al., 2016).

NAFLD is considered a multisystem disease and a hepatic manifestation of metabolic syndrome (Vernon et al., 2011; Gaudio et al., 2012). The published pediatric literature confirms that it is associated with a number of metabolic risk factors including dyslipidemia, insulin resistance, impaired glucose tolerance and visceral obesity (Temple et al., 2016; Marchesini et al., 2016; Vernon et al., 2011; Gaudio et al., 2012). Growing scientific evidence suggests that NAFLD is an independent risk factor for cardiovascular disease (CVD), whereas CVD remains one of the major causes of excess mortality in adult NAFLD patients (Targher et al., 2010; Söderberg et al., 2010).

The precise pathophysiological mechanism linking NAFLD and cardiovascular complications remains unclear. In fact, children with NAFLD display increased free fatty acids levels that may lead to myocardial lipid accumulation with consequent impairments in myocardial substrate metabolism and efficiency. Moreover, the liver is responsible for increased production of very low density lipoproteins (VLDL), which results in high concentrations of small dense LDL (sdLDL) in plasma and accumulation in plaques leading to ischemic events and high systolic blood pressure associated with NAFLD which in turn may affect myocardial structure, metabolism and function (Di Sessa et al., 2017).

Recent studies have also shown that visceral adipose tissue is the main source of hepatic fat and accomplishes important endocrine functions (Lebensztejn et al., 2016). It produces numerous biologically active substances including pro-inflammatory adipokines such as: tumor necrosis factor- $\alpha$ (TNF- $\alpha$ ), interleukin-6 (IL-6), visfatin and leptin, which promote local inflammation and progression to non-alcoholic steatohepatitis (NASH) and cirrhosis. Circulating adipokines also appear to trigger a systemic inflammatory response and induce oxidative 
stress which promotes early atherosclerosis. In contrast, adiponectin seems to be decreased in patients with NAFLD with low adiponectin levels possibly affecting cardiac function and atherogenic risk (Di Sessa et al., 2017). What is more, experimental and human studies point out the role of hepatokines released mainly from the liver, such as fetuin A, fibroblast growth factor-21 (FGF-21) and selenoprotein $\mathrm{P}$, in affecting the metabolism of lipids and glucose, which initiates pathogenic pathways potentially contributing to a pro-atherogenic environment. Importantly, FGF-21 is a protein synthesized and secreted by the liver in high amounts in obese subjects with a fatty liver and some investigators have speculated it might play a role in cardiac hypertrophy (Lebensztejn et al., 2016).

Coronary artery disease and stroke usually occur in middle and late age, but autopsy studies have demonstrated that the atherosclerotic process in the vascular wall starts in childhood and accelerates with exposure to risk factors (Pacifico et al., 2011).

Most studies concentrate on sophisticated methods to assess the cardiovascular system in obese patients. Data about the use of noninvasive biochemical markers useful in everyday practice is limited. The results of adults studies suggest that GGT may be identified as an independent biomarker of increased cardiovascular risk (Ndreppa \& Kastrati, 2016; Lonardo \& Romagnoli, 2016; Kasapoglu et al., 2016; Arinc et al., 2013). Therefore, the aim of this study was to ascertain if GGT can be regarded as a simple biomarker of cardiovascular risk in obese children with NAFLD.

\section{MATERIALS AND METHODS}

This prospective study involved a group of one hundred obese children (BMI $>95$ pc, 32 girls and 68 boys) aged from 7 to 17 years (median: 13 years), admitted to our Department because of suspected liver pathology: hepatomegaly, ultrasonographic liver brightness or increased ALT activity in serum. The study protocol was approved by the local bioethics committee. The purpose of the study was carefully explained to the parents of the children who gave their written consent to participate.

All subjects underwent physical examination with anthropometric measurements, barefoot and wearing minimal garments. Body mass index (BMI) was calculated by dividing weight $(\mathrm{kg})$ by height squared $\left(\mathrm{m}^{2}\right)$. Waist circumference was measured according to WHO protocol taking a measurement at the approximate midpoint between the lower margin of the last palpable rib and the top of the iliac crest.

Other liver disorders connected with steatosis such as: viral (HBV, HCV), selected metabolic (Wilson's disease, alpha-1-antitrypsin deficiency, cystic fibrosis), autoimmune and toxic (drug induced liver injury - DILI) conditions were excluded in all patients. Additionally, children with diabetes or those receiving drugs that could affect lipid metabolism were excluded from the study.

Blood chemistry analyses: alanine aminotransferase (ALT), gamma-glutamyl transferase (GGT), total bilirubin, total cholesterol, high-density lipoprotein (HDL), low-density lipoprotein (LDL), triglycerides, fasting glucose, insulin, high-sensitive C-reactive protein (hSCRP), were measured after a minimum 12-hour fasting period. ALT and GGT activities were determined by kinetic method (Cobas 6000-c501, Roche Diagnostics USA) and a serum ALT level $>37$ IU/1 was considered abnormal. GGT values 9-40 IU/1 were considered normal.
Total bilirubin was estimated using the Diazo Special method (Cobas 6000-c501, Roche Diagnostics USA) and the upper limit was $1.2 \mathrm{mg} / \mathrm{dl}$.

Total cholesterol was assessed with an enzymatic, colorimetric method (Cobas 6000-c501, Roche Diagnostics USA) and values $120-200 \mathrm{mg} / \mathrm{dl}$ were considered normal.

HDL-cholesterol, LDL-cholesterol and triglycerides concentration were determined by a homogenous enzymatic colorimetric method (Cobas 6000-c501, Roche Diagnostics USA) and values $35-65 \mathrm{mg} / \mathrm{dl}$ for HDL-cholesterol, $<130 \mathrm{mg} / \mathrm{dl}$ for LDL-cholesterol and 35-150 $\mathrm{mg} / \mathrm{dl}$ for triglycerides were considered normal.

Glucose concentration was assessed with the hexokinase method (Cobas 6000-c501, Roche Diagnostics USA) and values $60-100 \mathrm{mg} / \mathrm{dl}$ were considered normal. Insulin concentration was measured by a chemiluminescent immunometric assay (Immulite 1000, Siemens).

HOMA-IR (homeostatic model assessment-insulin resistance) was calculated according to Matthews et al. (1985).

To evaluate serum CRP, a high sensitivity method was used (Latex-enhanced immunoturbidometric assay, Cobas 6000-c501, Roche Diagnostics USA).

Abdominal ultrasound (USG) was performed in all participants by the same, experienced radiologist, using a General Electric Voluson E8 with a convex 3-5 Mhz transducer. The degree of liver steatosis in USG was assessed according to a four-grade (0-3) scale by Saverymuttu et al. (1986).

Common carotid artery intima-media thickness (IMT) was measured on the right and left side, in the supine position with the neck extended and the chin turned away from the side being examined, also by the same radiologist in all cases. Measurements were calculated on three end diastolic images and averaged.

${ }^{1}$ HMRS was performed with $1.5 \mathrm{~T}$ scanner (Picker Eclipse) and with PRESS sequencing. Total intrahepatic lipid concentration was assessed in relative units (r.u.) in comparison to an unsuppressed water signal (Tarasów et al., 2002).

Statistical analysis. Biochemical tests and anthropometric parameters were expressed as median and 25-75 quartile (Q1-Q3). Statistical analysis was performed using Statistica v 10.0. The Mann-Whitney test was used for non-parametric data. The relationship between biochemical tests was analyzed by the Spearman rank-correlation test. Results were considered statistically significant at $p<0.05$.

IBM SPSS Statistics 20.0 was used to analyze logistic regression.

Receiver operating characteristics (ROC) analysis was performed to calculate the power of the test to detect children with NAFLD.

\section{RESULTS}

\section{Characteristics of the examined group of children}

Characteristics of the examined group of children is presented in Table 1. NAFLD was confirmed in 38 out of the 100 obese patients enrolled in this study who had liver steatosis on USG examination and simultaneously increased serum ALT activity. Obese children who had neither liver steatosis on USG nor increased ALT activity were taken as the control group (obese non-hepatopathic children, $\mathrm{n}=18$ ). 
Table 1. Characteristics of the examined group of children $(n=100)$

\begin{tabular}{|c|c|c|c|}
\hline Parameter & Median & Q1 & Q3 \\
\hline Age (years) & 13 & 11 & 15 \\
\hline BMI $\left(\mathrm{kg} / \mathrm{m}^{2}\right)$ & 28 & 26.2 & 32.2 \\
\hline Waist $(\mathrm{cm})$ & 95 & 90 & 105.5 \\
\hline ALT (IU/I) & 34 & 21 & 57 \\
\hline GGT (IU/I) & 21 & 15 & 30 \\
\hline Bilirubin (mg/dl) & 0.6 & 0.5 & 0.8 \\
\hline Total cholesterol (mg/dl) & 170.5 & 144 & 189.5 \\
\hline HDL-cholesterol $(\mathrm{mg} / \mathrm{dl})$ & 48 & 40 & 55 \\
\hline LDL-cholesterol (mg/dl) & 93.5 & 76 & 116 \\
\hline Triglycerides (mg/dl) & 107 & 77 & 152.5 \\
\hline Glucose (mg/dl) & 90 & 83 & 95 \\
\hline Insulin $(\mu \mathrm{IU} / \mathrm{ml})$ & 14.9 & 11.6 & 19.5 \\
\hline HOMA-IR & 3.46 & 2.4 & 4.4 \\
\hline hsCRP (mg/l) & 1.2 & 0.7 & 2.0 \\
\hline USG - steatosis grade & 1 & 1 & 2 \\
\hline Right IMT (mm) & 0.52 & 0.48 & 0.57 \\
\hline Left IMT (mm) & 0.52 & 0.48 & 0.57 \\
\hline Mean IMT $(\mathrm{mm})$ & 0.52 & 0.48 & 0.57 \\
\hline Lipids 1HMRS (r.u.) & 103 & 55 & 170 \\
\hline
\end{tabular}

Table 2. Comparative characteristics of obese children with NAFLD and obese non-hepatopathic children

\begin{tabular}{|c|c|c|c|}
\hline Parameter & $\begin{array}{l}\text { Children with NAFLD } \\
(\mathrm{n}=38) \\
\text { Median; Q1-Q3 }\end{array}$ & $\begin{array}{l}\text { Obese non-hepatopathic children } \\
(\mathrm{n}=18) \\
\text { Median; Q1-Q3 }\end{array}$ & $P$ \\
\hline Age (years) & $14 ; 12-16$ & $12 ; 11-14$ & NS \\
\hline $\mathrm{BMI}(\mathrm{kg} / \mathrm{m} 2)$ & $30.1 ; 27.2-33.5$ & $28.7 ; 25.4-30.3$ & NS \\
\hline Waist $(\mathrm{cm})$ & $101 ; 94.5-107.5$ & $85.5 ; 81-98$ & 0.014 \\
\hline ALT (IU/I) & $66.5 ; 51-95$ & $18 ; 15-28$ & 0.0000 \\
\hline GGT (IU/I) & $30.5 ; 22-45$ & $14.5 ; 12-23$ & 0.000013 \\
\hline Bilirubin (mg/dl) & $0.7 ; 0.5-0.9$ & $0.6 ; 0.5-0.8$ & NS \\
\hline Total cholesterol (mg/dl) & $178.5 ; 151-214$ & $148.5 ; 137-178$ & 0.025 \\
\hline HDL-cholesterol (mg/dl) & $45.5 ; 40-54$ & $48 ; 42-58$ & NS \\
\hline LDL-cholesterol (mg/dl) & $95 ; 79-129$ & $80.5 ; 68-100$ & 0.046 \\
\hline Triglycerides $(\mathrm{mg} / \mathrm{dl})$ & $110.5 ; 76-167$ & $87.5 ; 61-136$ & NS \\
\hline Glucose (mg/dl) & $91 ; 81-97$ & $85 ; 81-91$ & NS \\
\hline Insulin $(\mu \mathrm{IU} / \mathrm{ml})$ & $16.7 ; 12.8-21$ & $14.5 ; 9.6-18$ & NS \\
\hline HOMA-IR & $3.8 ; 2.8-4.7$ & $3.2 ; 2.1-3.9$ & NS \\
\hline $\mathrm{hsCRP}(\mathrm{mg} / \mathrm{l})$ & $0.9 ; 0.7-1.8$ & $1.1 ; 0.7-2.2$ & NS \\
\hline Right IMT (mm) & $0.52 ; 0.5-0.57$ & $0.48 ; 0.42-0.54$ & NS \\
\hline Left IMT (mm) & $0.54 ; 0.51-0.58$ & $0.49 ; 0.46-0.52$ & 0.02 \\
\hline Mean IMT (mm) & $0.53 ; 0.5-56$ & $0.48 ; 0.44-0.55$ & 0.05 \\
\hline Lipids 'HMRS (r.u.) & $145 ; 95-210$ & $39 ; 17-61$ & 0.000004 \\
\hline
\end{tabular}


Table 3. Logistic regression analysis differentiating NAFLD patients from obese children without NAFLD

\begin{tabular}{|c|c|c|c|}
\hline Parameter & OR & $95 \% \mathrm{Cl}$ & $\mathrm{P}$ \\
\hline Waist (cm) & 1.209 & $1.034-1.414$ & 0.017 \\
\hline GGT (IU/I) & 1.069 & $1.01-1.131$ & 0.021 \\
\hline Total cholesterol $(\mathrm{mg} / \mathrm{dl})$ & 1.019 & $1.001-1.037$ & 0.038 \\
\hline HDL-cholesterol (mg/dl) & 0.981 & $0.933-1.031$ & NS \\
\hline LDL-cholesterol (mg/dl) & 1.022 & $1.000-1.043$ & 0.045 \\
\hline Triglycerides (mg/dl) & 1.009 & $0.998-1.02$ & NS \\
\hline HOMA-IR & 1.118 & $0.851-1.469$ & NS \\
\hline hsCRP (mg/l) & 0.855 & $0.411-1.776$ & NS \\
\hline Right IMT* $(\mathrm{mm})$ & 3.429 & $0.673-17.459$ & NS \\
\hline Left IMT* (mm) & 26.52 & $1.28-549.594$ & 0.034 \\
\hline Mean IMT* $(\mathrm{mm})$ & 0.313 & $1.08-153.682$ & NS \\
\hline
\end{tabular}

*OR and $\mathrm{Cl}$ displayed in conversion to 0.1 unit

Table 4. Ability of GGT, waist circumference and left IMT to detect children with NAFLD

\begin{tabular}{lllllc}
\hline Parameter & AUC & p & Se & Sp & NPV \\
\hline GGT & 0.94 & 0.000 & $94.12 \%$ & $83.33 \%$ & $83.33 \%$ \\
\hline Waist & 0.83 & 0.002 & $94.12 \%$ & $66.67 \%$ & $80 \%$ \\
\hdashline Left IMT & 0.82 & 0.002 & $88.24 \%$ & $66.67 \%$ & $88.89 \%$ \\
\hline
\end{tabular}

Liver steatosis was confirmed in 82 patients using abdominal USG and in 75 children using ${ }^{1}$ HMRS.

There was a significantly higher activity of GGT and ALT, concentration of total cholesterol, LDL-cholesterol, waist circumference and total lipids in ${ }^{1} \mathrm{HMRS}$ in individuals with NAFLD in comparison to non-hepatopathic obese children. Also, left coronary artery-IMT and mean IMT were higher in the NAFLD group than in non-hepatopathic obese children (Table 2).

Correlation of GGT activity in patients with traditional cardiovascular risk factors was weak: glucose level $(\mathrm{r}=0.2 ; p=0.04)$, waist circumference $(\mathrm{r}=0.36 ; p=0.01)$ and BMI ( $\mathrm{r}=0.24 ; p=0.02)$ or at the upper limit of statistical significance: total cholesterol $(\mathrm{r}=0.19 ; p=0.05)$, LDL-cholesterol $(\mathrm{r}=0.2 ; p=0.05)$ and insulin level $(\mathrm{r}=0.19 ; p=0.05)$.

The results of logistic regression analysis demonstrated that GGT, total cholesterol, LDL-cholesterol, left IMT and waist circumference were significant parameters differentiating NAFLD patients from non-hepatopathic obese children (Table 3). In the ROC analysis, among different anthropometric and biochemical parameters only GGT, waist circumference and left IMT presented the differentiation capacity for both conditions with the AUC for GGT being the highest (Figs 1, 2, 3, Table 4).

The combination of these markers (GGT, waist circumference, left IMT) was not superior.

\section{DISCUSSION}

In the presented study we found that GGT, waist circumference and left IMT were the best tests to differentiate children with NAFLD from obese, nonhepatopathic patients. Among the three parameters, GGT seems to be the most accurate, since the AUC for GGT was the highest in the ROC analysis. Furthermore, GGT activity in our patients correlated with traditional risk factors for cardiovascular disease (total cholesterol,
LDL-cholesterol, glucose, insulin, waist circumference and BMI). Therefore, GGT may be regarded as a suitable simple, non-invasive biochemical marker of cardiovascular risk in children with NAFLD.

Ample evidence suggests that increased GGT activity is a marker of antioxidant inadequacy and increased oxidative stress. The presence of GGT activity in atherosclerotic plaques and correlation of intra-plaque GGT activity with histological indexes of plaque instability may support the theory of GGT

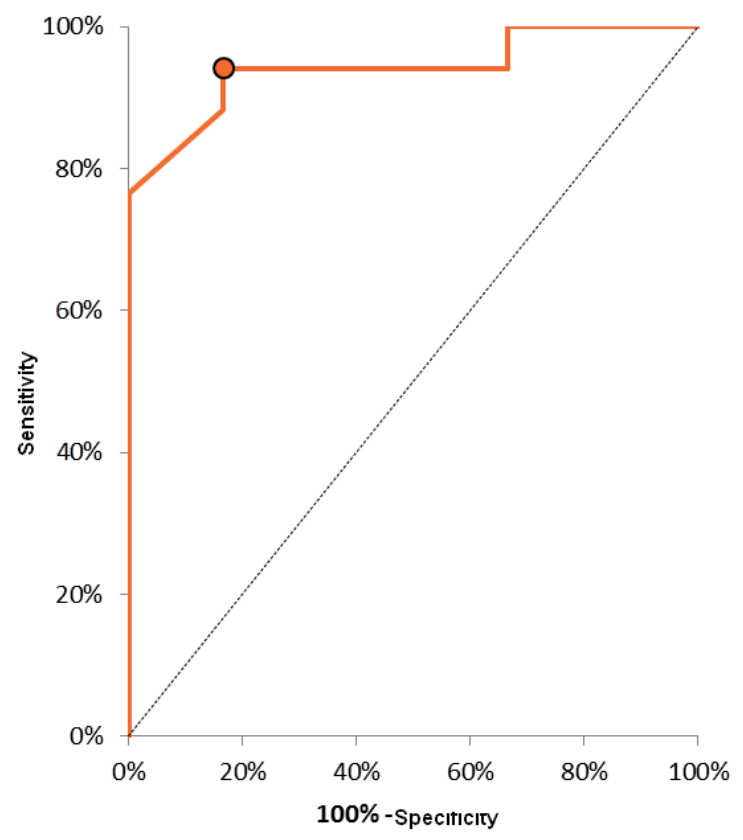

Figure 1. Receiver operating characteristics (ROC) curve of ability of GGT to detect children with NAFLD (cut off $>19.5$ ). 


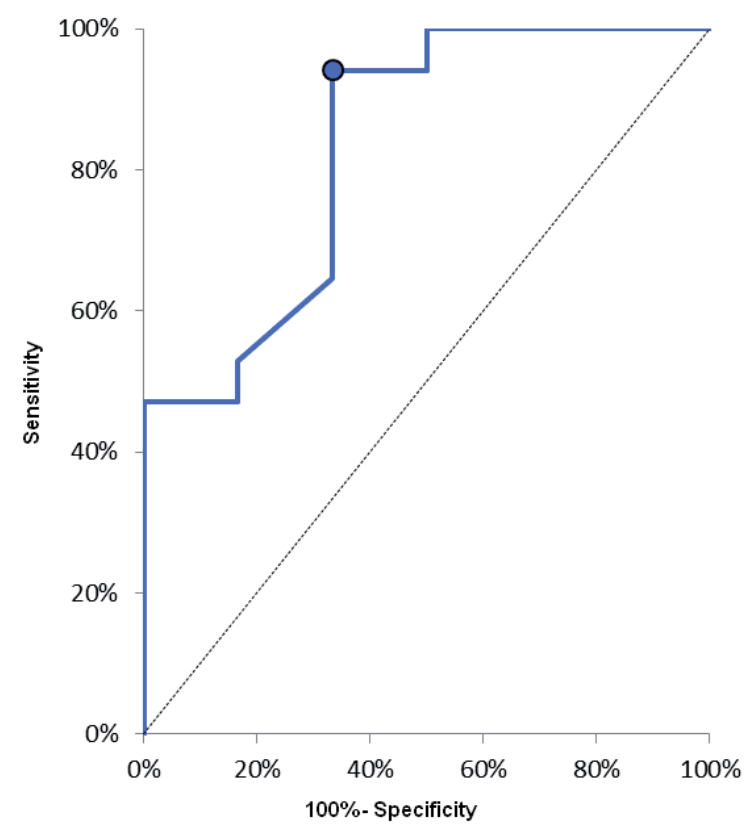

Figure 2. Receiver operating characteristics (ROC) curve of ability of waist circumference to detect children with NAFLD (cut off $>91$ ).

involvement in the pathophysiology of CVD, particularly atherosclerosis (Ndreppa \& Kastrati, 2016; Lonardo et al., 2016).

Kasapoglu and coworkers (Kasapoglu et al., 2016) in their large, but retrospective study (982 control individuals vs 1818 individuals with various degrees of fatty liver evaluated ultrasonographically) found a positive correlation between elevated serum GGT levels and the Framingham cardiovascular scoring system among non-diabetic, non-obese adults with NAFLD. The risk of cardiovascular disease increased with the presence and severity of fatty liver disease. The authors concluded that high GGT levels among patients with fatty liver disease should be regarded as an alarm sign of increased cardiovascular risk.

Arinc and coworkers (Arinc et al., 2013), in a study conducted in 50 adult patients with biopsy-proven NASH, also found GGT to be a predictor of endothelial dysfunction. The authors measured brachial artery FMD (flow-mediated dilatation) and CIMT (carotid artery intima-media thickness) as markers of endothelial function. Patients with NASH had impaired FMD and increased CIMT when compared to healthy controls. Linear regression analysis showed that serum GGT concentration was significantly associated with FMD and CIMT.

The role of GGT in predicting cardiovascular risk in children remains under-explored. Kong and coworkers (Kong et al., 2013) found that GGT and uric acid have a combined effect in association with obesity and cardiovascular risk factors (obesity, low HDL cholesterol (HDL-C) level and high blood pressure) in children and adolescents.

Serum GGT was also positively associated with metabolic syndrome and its components in Korean children and adolescents (Park et al., 2017; Cho et al., 2011).

Our study is also in agreement with the study by Alkhouri and coworkers (Alkhouri et al., 2014) conducted in children with biopsy-proven NAFLD. The significance of non-HDL-C as a distinguishing marker for NASH on multivariate analysis was lost when controlling for such

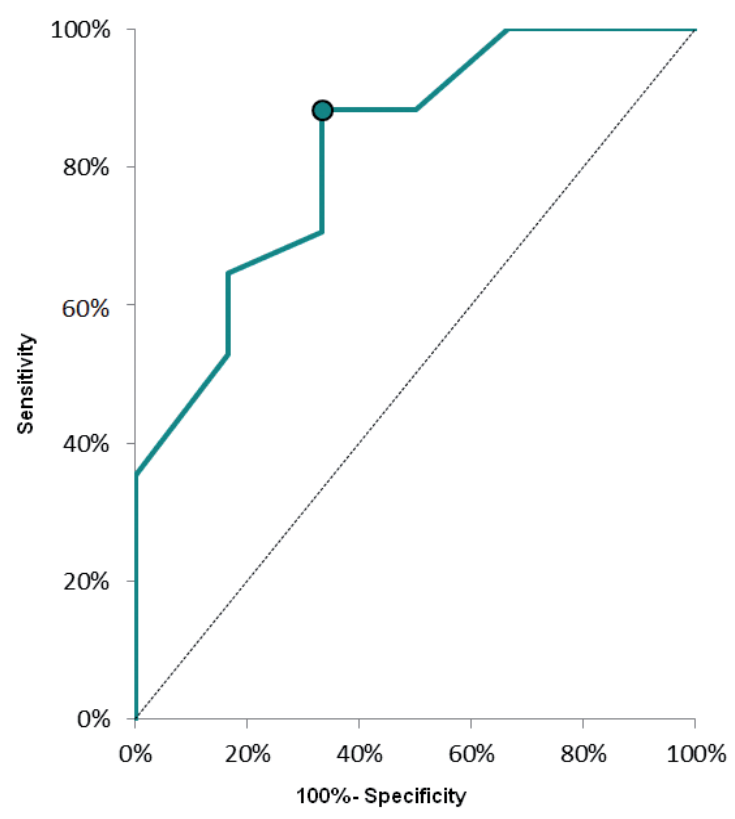

Figure 3. Receiver operating characteristics (ROC) curve of ability of left IMT to detect children with NAFLD. (cut off $>0.5$ ).

serum markers as GGT and ALT, which themselves are markers of inflammation and are associated with cardiovascular risk.

Nevertheless, the main objective in our study was to determine which of the examined parameters can be considered as the best non-invasive, simple and valuable marker of CVD in children. Yang and Chang (Yang \& Chang, 2016) did not find conventional anthropometry helpful in differentiating obese children with and without NAFLD and adopted a more advanced method - dual energy X-ray absorptiometry (DXA). We found waist circumference very simple and useful and our finding was supported by other authors (Yoo et al., 2008; Motamed et al., 2016). However, the result may be less accurate if the measurement is not performed properly. In contrast, GGT is always objective as it is assessed in an automated analyzer.

Furthermore, IMT was higher only in the left coronary artery in patients with NAFLD in our study. The IMT on the right side was comparable in both NAFLD patients and in controls. Manco and coworkers (Manco et al., 2010) found a higher left IMT in patients with NAFLD detected with ultrasonography and confirmed by liver biopsy. Although statistically significant, this difference is unlikely to be clinically relevant because of a substantial overlap of CIMT values between the cases and controls. The authors did not confirm an association between CIMT and the histological severity of the steatosis and fibrosis.

In contrast, Man and coworkers (Man et al., 2017) measured carotid IMT in obese adolescents and in lean controls and found this parameter significantly greater in obese subjects. Moreover, univariate analyses showed positive correlation between serum ALT and alkaline phosphatase levels and carotid IMT.

Madan and coworkers (Madan et al., 2015) carried out a meta-analysis to evaluate the relationship between NAFLD and carotid atherosclerosis measured as CIMT. Their analysis showed that NAFLD is associated with increased CIMT in both children and adults. In conclu- 
sion, the role of CIMT as a marker of CVD in children is not clear. The procedure also requires an experienced radiologist whereas GGT assessment is very simple and widely available.

Our work has several potential limitations. Firstly, the number of patients was relatively too small to draw definite conclusions. Secondly, the absence of a biopsy regarded as the 'gold standard' in diagnosis of NAFLD/ NASH, limits the generalizability of our findings. However, the procedure is invasive and the examined children in our study did not meet the criteria to perform a liver biopsy (Dezsofi et al., 2015).

In conclusion, the obtained data allowed us to suggest that GGT might be a reliable, simple and non-invasive biochemical marker for estimation of cardiovascular risk in obese children with NAFLD. However, further studies on larger population are necessary to confirm that observation.

\section{Acknowledgements o Financial Support}

This study was financially supported by grant No 14343618L from the Medical University in Bialystok, Poland.

\section{REFERENCES}

Alkhouri N, Eng K, Lopez R, Nobili V (2014) Non-high-density lipoprotein cholesterol (non-HDL-C) levels in children with nonalcoholic fatty liver disease (NAFLD). Springerplus 3: 407. http://doi. org/10.1186/2193-1801-3-407

Arinc H, Sarli B, Baktir AO, Saglam H, Demirci E, Dogan Y, Kurtul S, Karaman H, Erden A, Karaman A (2013) Serum gamma glutamyl transferase and alanine transaminase concentrations predict endothelial dysfunction in patients with non-alcoholic steatohepatitis. Ups J Med Sci 118: 228-234. http://doi:10.3109/03009734.2013.814734

Cho YG, Park KH, Kim CW, Hur YI (2011) The relationship between serum gamma-glutamyl transferase level and overweight in Korean urban children. Korean J Fam Med 32: 182-188. http://doi:10.4082/ kjfm.2011.32.3.182

Dezsofi A, Baumann U, Dhawan A, Durmaz O, Fischler B, Hadzic N, Hierro L, Lacaille F, McLin VA, Nobili V, Socha P, Vajro $\mathrm{P}$, Knisely AS (2015) Liver biopsy in children: position paper of the ESPGHAN Hepatology Committee, JPGN 60: 408-420. http:// doi:10.1097/MPG.0000000000000632

Di Sessa A, Umano GR, del Giudice EM, Santoro N (2017) From the liver to the heart: Cardiac disfunction in obese children with non-alcoholic fatty liver disease. World J Hepatol 9: 69-73. http:// doi:10.4254/wjh.v9.i2.69

Gaudio E, Nobili V, Franchitto A, Onori P, Carpino G (2012) Nonalcoholic fatty liver disease and atherosclerosis. Intern Emerg Med 7: 297-305. http:/doi: 10.1007/s11739-012-0826-5

Kasapoglu B, Turkay C, Yalcin KS, Carlioglu A, Koktener A (2016) Role of $\gamma$-glutamyl transferase levels in prediction of high cardiovascular risk among patients with non-alcoholic fatty liver disease, Indian J Med Res 143: 30-36. http://doi:10.4103/0971-5916.178585

Kong AP, Choi KC, Ho CS, Chan MH, Ozaki R, Chan CW, Chan JC (2013) Associations of uric acid and gamma-glutamyltransferase (GGT) with obesity and components of metabolic syndrome in children and adolescents. Pediatr Obes 8: 351-357. http// doi:10.1111/j.2047-6310.2012.00115.x

Lebensztejn D, Flisiak-Jackiewicz M, Białokoz-Kalinowska I, BobrusChociej A, Kowalska I (2016) Hepatokines and non-alcoholic fatty liver disease Acta Biochim Pol 63: 459-467. http://doi:10.18388/ abp.2016_1252

Lonardo A, Romagnoli D: Gamma-glutamyl transferase: a novel cardiovascular outfit for an old liver test (2016). Indian J Med Res 143: 4-7. http://doi:10.4103/0971-5916.178574

Madan SA, John F, Pyrsopoulos N, Pitchumoni CS (2015) Nonalcoholic fatty liver disease and carotid artery atherosclerosis in children and adults: a meta-analysis. Eur J Gastroenterol Hepatol 27: 1237-1248. http://doi:10.1097/MEG.0000000000000429

Man E, Cheung PT, Cheung YF (2017) Associations between arterial structure and function and serum levels of liver enzymes in obese adolescents. J Paediatr Child Health 53: 691-697. http://doi: 10.1111/jpc. 13528

Marchesini G, Day ChP, Dufour JF, Canbay A, Nobili V, Ratziu V, Tilg H, Roden M, Gastaldelli A, Yki-Järvinen H, Schick F, Vettor R, Frühbeck G, Mathus-Vliegen L (2016) EASL-EASD-EASO Clinical Practice Guidelines for the management of non-alcoholic fatty liver disease. J Hepatol 64: 1388-1402. http://doi: 10.1016/j. jhep.2015.11.004

Manco M, Bedogni G, Monti L, Morino G, Natali G, Nobili V (2010) Intima media thickness and liver histology in obese children and adolescents with NAFLD. Atherosclerosis 209: 463-468. https://doi. org/10.1016/j.atherosclerosis.2009.10.014

Matthews DR, Hosker JP, Rudenski AS, Naylor BA, Treacher DF, Turner RC (1985) HOMA-IR and beta-cell function from fasting plasma glucose and insulin concentration in man. Diabetologia 28: 412-419

Motamed N, Sohrabi M, Ajdarkosh H, Hemmasi G, Maadi M, Sayeedian FS, Pirzad R, Abedi K, Aghapour S, Fallahnezhad M, Zamani F (2016) Fatty liver index vs waist circumference for predicting non-alcoholic fatty liver disease. World J Gastroenterol 14: 3023-3030. http://doi: 10.3748/wig.v22.i10.3023

Ndreppa G, Kastrati A (2016) Gamma-glutamyl transferase and cardiovascular disease. Ann Transl Med 4: 481. http://doi:10.21037/ atm.2016.12.27

Pacifico L, Nobili V, Anania C, Verdecchia P, Chiesa C (2011) Pediatric nonalcoholic fatty liver disease, metabolic syndrome and cardiovascular risk. World J Gastroenterol 17: 3082-3091. http://doi: 10.3748/wig.v17.i26.3082

Park JM, Lee JY, Lee DC, Lee YJ (2017) Serum $\gamma$-glutamyltransferase level and metabolic syndrome in children and adolescents: Korean National Health and Nutrition Examination Survey. I Diabetes Investig 1-7. http://doi:10.1111/jdi.12716

Saverymuttu SH, Joseph AE, Maxwell JD (1986) Ultrasound scanning in the detection of hepatic fibrosis and steatosis. Br Med J 292: 1315

Söderberg C, Stål P, Askling J, Glaumann H, Lindberg G, Marmur J, Hultcrantz R (2010) Decrease survival of subjects with elevated liver function tests during a 28-year follow up. Hepatology 51: 595-602. http://doi:10.1002/hep.23314

Tarasów E, Siergiejczyk L, Panasiuk A, Kubas B, Dzienis W, Prokopowicz D, Walecki J (2002) MR proton spectroscopy in liver examination of healthy individuals in vivo. Med Sci Monit 8: 36-40

Targher G, Day CP, Bonora E (2010) Risk of cardiovascular disease in patients with non- alcoholic fatty liver disease. $N$ Eng J Med 363: 1341-1350. http://doi:10.1056/NEJMra0912063

Temple JL, Cordero P, Li J, Nguyen V, Oben JA (2016) A guide to non-alcoholic fatty liver disease in childhood and adolescence. Int $J$ Mol Sci 17: 947. https://doi.org/10.3390/ijms17060947

Vernon G, Baranova A, Younossi ZM (2011) Systematic review: the epidemiology and natural history of non-alcoholic fatty liver disease and non-alcoholic steatohepatitis in adults. Aliment Pharmacol Ther 34: 274-285. http://doi:10.1111/j.1365-2036.2011.04724.x

Yang HR, Chang EJ (2016) Insulin resistance, body composition and fat distribution in obese children with nonalcoholic fatty liver disease. Asia Pac J Clin Nutr 25: 126-133. http://doi:10.6133/apjcn.2016.25.1.15

Yoo J, Lee SY, Kim KN, Yoo SM, Sung EJ, Yim JE (2008) Relationship between insulin resistance and serum alanine aminotransferase as a surrogate of NAFLD (nonalcoholic fatty liver disease) in obese Korean children. Diabetes Res Clin Pract 81: 321-326. https://doi. org/10.1016/j.diabres.2008.05.006 